\title{
Análisis de la Medición de la Biomasa en Fermentación en Estado Sólido empleando el Modelo Logístico y Redes Neuronales
}

\author{
Juan C. Oviedo(1), Ana E Casas ${ }^{(2)}$, Jaime A Valencia ${ }^{(3)}$ y José E. Zapata(4) \\ (1) Escuela de Ingenierías, Facultad de Ingeniería Agroindustrial, Universidad Pontificia Bolivariana, \\ Circular 1 No. 70-01, Medellín-Colombia, (e-mail: juan.oviedo@upb.edu.co). \\ (2) Escuela de Ingenierías, Facultad de Ingeniería Textil, Universidad Pontificia Bolivariana, Circular 1 No. \\ 70-01 Medellín-Colombia, (e-mail: ana.casas@upb.edu.co) \\ (3) Facultad de Ingeniería, Departamento Ingeniería Eléctrica, UdeA, Calle 67 No. 53 -108, \\ Medellín-Colombia (e-mail: valenciajaime1@gmail.com) \\ (4) Facultad de Química Farmacéutica, Departamento de Alimentos, UdeA. Calle 67 No. 53 - 108, \\ Medellín-Colombia. (e-mail: jegar_4@yahoo.es)
}

Recibido Dic. 16, 2013; Aceptado Feb. 25, 2014; Versión final recibida Mar. 30, 2014

\begin{abstract}
Resumen
En este trabajo, se llevó a cabo el análisis del crecimiento del Pleurotus pulmonarius en tusa de maíz, empleando redes neuronales artificiales y el modelo logístico. La biomasa se cuantificó a través de las concentraciones de proteína (método Kjeldahl) y ergosterol (cromatografía líquida de alta resolución). Los datos obtenidos fueron analizados con los programas $\mathrm{R}$ y Matlab. El mejor $\mathrm{r}^{2}$ ajustado del modelo logístico fue de 0,9937 en la concentración de proteína para el análisis ensayo por ensayo. Las redes neuronales artificiales, obtuvieron sumatorias medias de los errores cuadráticos de 0,017 y 11,394 para las concentraciones de proteína y ergosterol respectivamente. Los resultados muestran que el modelo logístico y las redes neuronales artificiales son herramientas útiles para modelar en fermentación sólida. Los mejores ajustes fueron para la concentración de proteína.
\end{abstract}

Palabras clave: fes, rna, biomasa, modelo logístico, Pleurotus pulmonarius

\section{Analysis of Biomass Measurement in Solid-State Fermentation using Neural Networks and a Logistic Model}

\begin{abstract}
In this work, the analysis of the growth of Pleurotus pulmonarius in corn cob, using artificial neural networks and a logistic model was carried out. Biomass is quantified through the concentrations of protein (Kjeldahl method) and ergosterol (High performance liquid chromatography). The data obtained were analyzed with the Matlab and R programs. The best adjusted $r^{2}$ of the logistic model was 0.9937 in the concentration for test by test protein analysis. For the artificial neural network model the root mean square error was 0.017 for the concentrations of protein and 11.394 for ergosterol. The results show that the logistic model and the artificial neural network model are useful tools for modeling solid fermentation. The best results were found for the concentration of protein.
\end{abstract}

Keywords: ssf, ann, biomass, logistic model, Pleurotus pulmonarius. 


\section{INTRODUCCIÓN}

La fermentación en estado sólido de forma general (FES), se puede definir como el crecimiento de microorganismos en un material sólido con ausencia o casi ausencia de humedad libre (Padey et al., 2008). El material sólido, sirve como soporte y fuente de nutrientes (Rodríguez et al., 2006; Hölter et al., 2005). Algunos de los procesos más importantes en la FES son el compostaje, ensilaje, producción de enzimas, sabores, ácidos orgánicos, producción de alimentos, combustibles, producción de metabolitos secundarios, producción de hongos comestibles, producción alimentos para animales, la degradación de tintas de la industria papelera entre otros (Robinson et al., 2003; Rodriguez et al., 2006; Carabajal et al., 2012). Lo anterior, muestra que la FES tiene unas ventajas, dentro de la que se destacan la alta productividad volumétrica, menor generación de efluentes y residuos, aprovechamiento de numerosos desperdicios agroindustriales, menores costos de energía en los procesos (Padey et al, 2008; Rodríguez et al., 2006; Robinson et al., 2003) entre otros. Sin embargo presenta como desventajas, dificultades para el escalado, baja eficiencia en la transferencia de masa y de calor, y dificultades en el control de los parámetros del proceso (Mitchell et al., 2006; Prabhakar et al., 2005; Muller et.al., 2004. Jenzsch, 2006). Adicional a lo anterior, la cuantificación de la biomasa y la modelación de su dinámica de crecimiento, son dos de los grandes retos de la FES (Rani et al., 2009). Se han establecido varios métodos para la medición de la biomasa por FES, como son la determinación de la producción de glucosamina, el contenido de ergosterol, la concentración proteína, cambios en el peso seco o evolución de la producción de $\mathrm{CO}_{2}$. Sin embargo, todos presentan inconvenientes a la hora de obtener datos precisos sobre el desarrollo del proceso de FES y la obtención de modelos matemáticos principalmente por la dispersión en el crecimiento de la célula sobre el sustrato solido (Thomas et al., 2013; Viccini et al., 2001; Padey et al., 2008). Se han llevado a cabo trabajos con el Pleurotus pulmonarius en FES, en diferentes sustratos agroindustriales, pero en tusa de maíz no se han encontrado reportes de medición de la biomasa en FES, ni su correspondiente modelación del crecimiento. Los resultados de las medidas de la biomasa, hace que la descripción matemática de la misma, se lleve a cabo a través de regresiones no lineales (Khaouane et al., 2013).

Dentro de las regresiones no lineales ésta el modelo logístico que ha sido empleado para describir la cinética de la biomasa en FES (Mitchel et al., 2004). El modelo de crecimiento logístico fue propuesto por Verhulst en 1838 y es un modelo que ilustra la dinámica de crecimiento para la mayoría de los organismos en un hábitat con recursos finitos; también es conocido como la función logística o curva en forma de S. El modelo logístico ha sido empleado para crecimiento de poblaciones, propagación de enfermedades, modelos de adopción agregada y la cuantificación de biomasa en bioprocesos tanto en sistemas líquidos (sumergidos) como sólidos (Quiñones et al., 2007; Orlandoni et al., 1997; Muñoz et al., 2011; Peleg et al., 2007). El comportamiento logístico, es uno de los cuatro perfiles básicos de crecimiento para FES, tal como lo describe Mitchell y colaboradores (2004). Por otro lado, están las RNA, que son estructuras formales de carácter matemático y estadístico, inspiradas en el comportamiento del cerebro humano y con la propiedad de aprender a partir de ejemplos (Sturm et al., 2008; Haykin, 2005; Villada et al., 2012). Las redes neuronales son una buena alternativa para modelar el crecimiento, debido a que está demostrado que pueden ajustarse a cualquier tipo de función, porque son considerados como aproximadores universales, es decir, que tienen la capacidad de acercarse a cualquier función matemática tanto como se desee, lo anterior se soporta en un teorema matemáticamente probado (Hayking, 2005). En particular, funciones sigmoideas, se ajustan fácilmente por que las neuronas tienen un parámetro relacionado con la función de activación que es una sigmoidea unitaria. La capacidad de aprender y de trabajar con sistemas no lineales, hacen que sean de interés para describir procesos como la FES (Sturm et al., 2008). Los objetivos de este trabajo son realizar un procesamiento estadístico de los datos de la biomasa del Pleurotus pulmonarius, medida a través de las concentraciones de proteína y ergosterol, empleando tusa de maíz como sustrato, y establecer la cinética de esta fermentación comparando los resultados con el modelo logístico y las RNA.

\section{MATERIALES Y MÉTODOS}

\section{Obtención y tratamiento de la tusa de maíz}

La tusa de maíz, se obtiene de pelar la mazorca en la Central Mayorista de Antioquia, ubicada en la ciudad de Itagüi. La mazorca es pelada y desgranada por los comercializadores de maíz. La tusa separada no tiene un tiempo superior a 8 horas, con el objetivo de reducir el riesgo de contaminación. Una vez obtenida, es llevada al laboratorio del Grupo de investigaciones agroindustriales, Grain, perteneciente a la Escuela de Ingenierías de la Universidad Pontificia Bolivariana, donde es desinfectada con una solución acuosa de hipoclorito de sodio, en una concentración de $5 \mathrm{~mL} / \mathrm{L}$ y luego es esterilizada a $121^{\circ} \mathrm{C}$ durante 30 minutos, en un autoclave. Posteriormente, es deshidratada en un horno de convección forzada a $75{ }^{\circ} \mathrm{C}$ hasta peso constante según la norma AOAC 925.10 (1997). Una vez seca, se lleva a un molino de cuchillas y se realiza un proceso de separación en un Rot-Tap por grupos de tamaño. El almacenamiento se realizó en bolsas de plástico sellables. 
Obtención de la semilla del Pleurotus pulmonarius e inoculo.

La semilla del basidiomiceto Pleurotus pulmonarius, se adquirió periódicamente en el laboratorio de Biotecnología Vegetal de la Universidad de Antioquia. Esta semilla era llevada a cajas de petri con agar PDA, (Agar Dextrosa y Papa), en cajas previamente esterilizadas a $121{ }^{\circ} \mathrm{C}$ durante 15 minutos. Las cajas de petri inoculadas son incubadas a $30^{\circ} \mathrm{C}$ durante 15 días en una cámara de incubación.

\section{Fermentación solida}

Las condiciones para la FES, se establecieron teniendo en cuenta los resultados preliminares obtenidos por Oviedo y colaboradores (2010). Dichas condiciones, arrojaron que el sustrato está compuesto así: En una caja de petri previamente esterilizada se adicionaron, $15 \mathrm{~g}$ de tusa tratada, $1 \%$ de $\mathrm{CaO}, 0,01 \% \mathrm{CuSO}_{4}{ }^{\star} 5 \mathrm{H}_{2} \mathrm{O}$ y agua de grifo del acueducto de Medellín, hasta un $80 \%$ de humedad del medio. El tamaño de partícula de la tusa fue el obtenido con los tamices entre 335 y 1170 micrómetros de la serie Tyler y a $25^{\circ} \mathrm{C}$. Las cajas de petri, con la mezcla anterior, fueron esterilizadas durante 20 minutos a $121^{\circ} \mathrm{C}$. Para la inoculación se emplearon trozos de aproximadamente de $1 \mathrm{~cm}^{2}$ del cultivo de Pleurotus pulmonarius en agar PDA. La inoculación se llevó a cabo en una cámara de flujo laminar previamente desinfectada con alcohol industrial. Las muestras se evaluaban cada tres días, debido a que en este mismo trabajo de Oviedo y colaboradores (2010) se determinó que era un intervalo de tiempo adecuado para hacer la observación del crecimiento. Finalmente, el proceso tuvo una duración de 30 días.

\section{Extracción y medición de la biomasa}

La medición de la biomasa, se llevó a cabo por los métodos indirectos de concentración de proteína y de ergosterol. Tanto para la concentración de la proteína y como de ergosterol, se llevó a cabo un proceso de extracción. En el caso de la proteína, la extracción se realizó por el método propuesto por Braaksma y colaboradores (1996), donde se hacen separaciones de las fracciones proteicas en frío con tampón de acetato y NaCl, acompañado de agitación, centrifugación y calentamiento. La cuantificación de la proteína se realizó por el método de Kjeldahl (AOAC, 1997) con un digestor y destilador automático DKL, de Velp Scientifica. La titulación (medición) se realizó con un potenciómetro Titrando 888, marca Metrohm.

Para el caso de la concentración de ergosterol, se empleó el método combinado de Brodie y colaboradores (2003) y Silva (2008). La extracción del ergosterol se realizó con una separación empleando KOH, etanol y metanol en agitación orbital durante 20 minutos; posteriormente se llevó a cabo una sonicación, en un equipo de ultra sonido marca Elma operado a 4,5 Amperios y $60 \mathrm{~Hz}$ a $40{ }^{\circ} \mathrm{C}$ durante 5 minutos; paso seguido se realizó una saponificación en un baño María Maxthermo operado a 120V y 6 Amperios a una temperatura de $75^{\circ} \mathrm{C}$ durante 35 minutos; luego se realizó una extracción con agua destilada-desionizada y hexano grado HPLC. Finalmente se centrifugó en un equipo Boeco Germany R: U320R a 7000 RPM durante 10 minutos. Terminado este paso el extracto de hexano fue llevado a la medición, en un HPLC que utilizó una columna C18 250x4.6mm, una fase móvil de metanol, con un flujo de $1.5 \mathrm{ml} / \mathrm{min}$ a una temperatura de $30^{\circ} \mathrm{C}$ y detección UV-VIS (280nm).

\section{Análisis de datos}

Con los datos obtenidos se construyeron las curvas cinéticas de biomasa para las concentraciones de proteína y ergosterol en Microsoft Excel®. Con el software R y el CFTOOL de Matlab®, se analizó el modelo logístico para las concentraciones de proteína y ergosterol. Para el análisis con RNA, el tipo de red empleada fue la denominada Backpropagation por su algoritmo de aprendizaje, también conocida como la red neuronal de propagación. Este tipo de RNA encuentra una relación funcional de los datos de entrada con respecto a los datos de salida, en este caso para encontrar un modelo de la cinética del crecimiento de biomasa. Para el procesamiento de las RNA se empleó el toolbox de redes neuronales de Matlab®, NFTOOL. EI NFTOOL por defecto, utilizó una configuración de dos capas: la primera, (capa oculta), emplea una función de activación sigmoidea; la otra capa, (de salida), emplea la función de activación lineal. Se seleccionaron cuatro neuronas en la capa oculta y una neurona en la capa de salida entrenándose hasta el ajuste.

\section{RESULTADOS Y DISCUSIÓN}

\section{Cinética de la biomasa}

La figura 1, muestra las medidas realizadas del crecimiento para las curvas de biomasa tanto para la concentración proteína por el método de Kjeldahl como para el contenido de ergosterol por HPLC. 

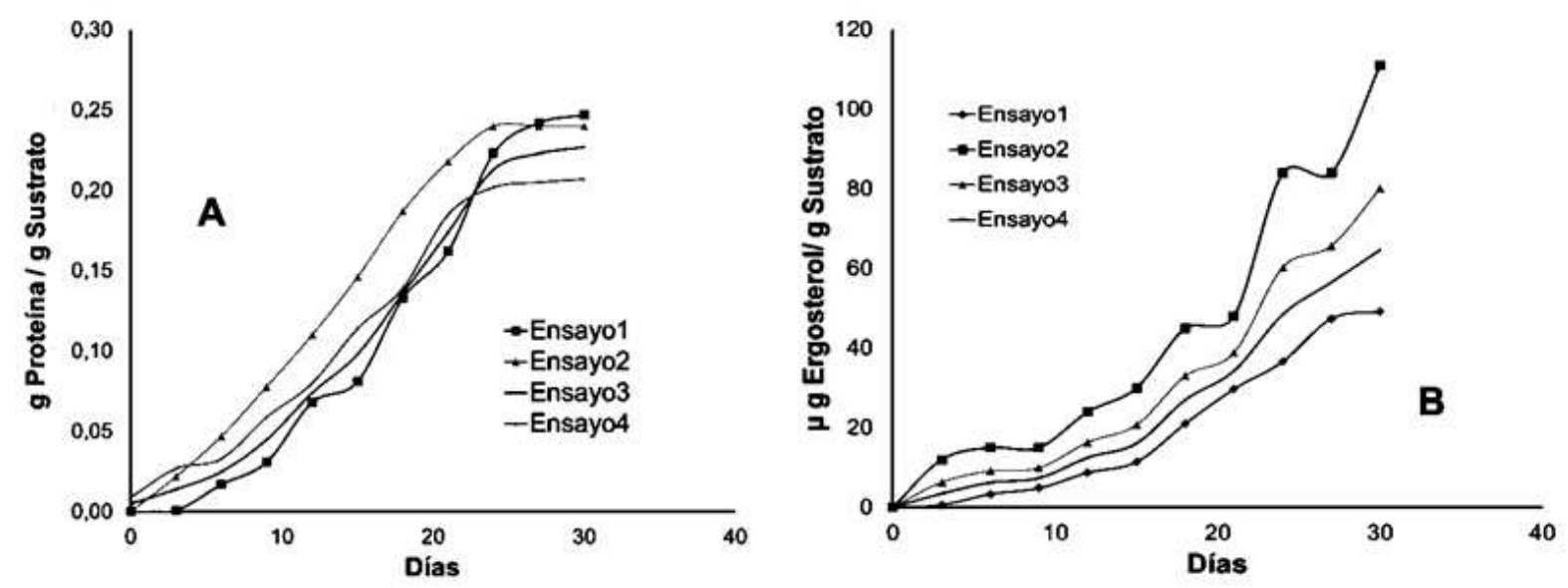

Fig. 1 Cinética de la biomasa del Pleurotus pulmonarius. A. Concentración de proteína; B. Concentración de ergosterol.

En el caso de la concentración de proteína, el máximo rendimiento para todos los experimentos se presentó a los 24 días; de allí en adelante la biomasa se estabilizó, debido a que se llegó a la fase estacionaria de crecimiento celular. El promedio del rendimiento máximo en las cuatro replicas fue de $0,230 \mathrm{~g}$ de Proteína/ $\mathrm{g}$ de Sustrato $\pm 0,0161$. Por su parte, en la concentración de ergosterol, el promedio del rendimiento máximo alcanzado fue de $76,238 \mu \mathrm{g}$ de Ergosterol/ $\mathrm{g}$ de Sustrato $\pm 26,3859$.

\section{Resultados del modelo logístico}

En ambos casos, la cinética de crecimiento muestra un comportamiento logístico, que entre algunas de sus características muestra una fase de estabilización y no cuenta con fase de adaptación (Vicchini et al., 2001). El modelo logístico se muestra en la ecuación 1

$x(t)=\frac{\beta 1}{1+e^{(\beta 2+\beta 3 * t)}}$

Donde $x$ es la concentración de biomasa que puede ser expresada en concentración de proteína ( $g$ de Proteína/g Sustrato) o la concentración de ergosterol ( $\mu \mathrm{g}$ de Ergosterol/g Sustrato); $\beta_{1} ; \beta_{2}$ y $\beta_{3}$ los coeficientes de la ecuación logística, que se obtienen mediante ajuste y $t$ el tiempo en días. El ajuste de los datos al modelo logístico para cada ensayo, con el software $R$ para las concentraciones de proteína y de ergosterol se muestra en la figura 2.
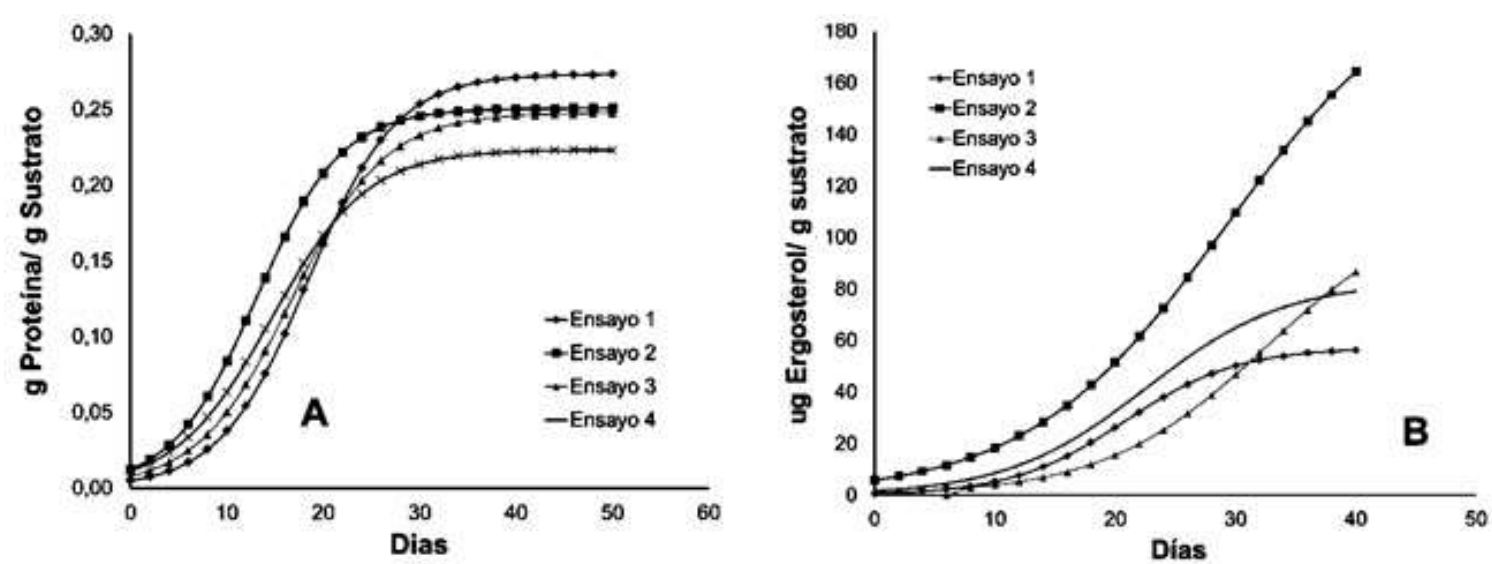

Fig. 2 Ajuste de la biomasa al modelo logístico para los cuatro ensayos. A. Concentración de proteína vs tiempo. B. Concentración de ergosterol vs tiempo

De los ajustes obtenidos en la figura 2, en la tabla 1 se muestran en forma resumida los coeficientes de la ecuación logística, para la concentración de proteína. 
Tabla 1. Coeficientes de la ecuación logística de la biomasa para la concentración de proteína vs tiempo

\begin{tabular}{|c|c|c|}
\hline Coeficientes & Valor Promedio & Desv. Estandar \\
\hline$\beta_{1}$ (g Proteína/g Sto.) & 0,2489 & 0,0204 \\
\hline$\beta_{2}$ & 3,3378 & 0,5113 \\
\hline$\beta_{3}$ (Dias $\left.^{-1}\right)$ & $-0,2131$ & 0,0121 \\
\hline$r^{2}$ ajustado & 0,9937 & - \\
\hline
\end{tabular}

Los valores mostrados en la tabla 1, indican un buen ajuste para el caso de la concentración de proteína al modelo logístico, con un valor $\mathrm{r}^{2}$ ajustado promedio de 0,9937. En cuanto a los coeficientes, de los ensayos, todos mostraron una desviación estándar baja, indicando poca dispersión en los valores. El coeficiente $\beta_{1}$, es un indicador de la máxima concentración de biomasa que se podría obtener, en este caso el valor promedio es $0,2489 \mathrm{~g}$ Proteína/ g Sustrato $\pm 0,0204$. El parámetro $\beta_{2}$ es adimensional y $\beta_{3}$ debe estar expresado en días ${ }^{-1}$. Los coeficientes promedio para la ecuación logística obtenidos para la concentración de ergosterol, se muestran en la tabla 2.

Tabla 2. Coeficientes de la ecuación logística en la biomasa para la concentración de ergosterol vs tiempo

\begin{tabular}{|c|c|c|}
\hline Coeficientes & Valor Promedio & Desv. Estandar \\
\hline$\beta_{1}\left(\right.$ g Proteína/ g Sto.) $^{\prime}$ & 116,146 & 65,8616 \\
\hline$\beta_{2}$ & 3,8661 & 0,3845 \\
\hline$\beta_{3}$ (Dias $\left.^{-1}\right)$ & $-0,1633$ & 0,0384 \\
\hline r $^{2}$ Ajustado & 0,9872 & - \\
\hline
\end{tabular}

Para el caso de la concentración de ergosterol, se obtuvo un $\mathrm{r}^{2}$ promedio de 0,9872 ; dicho ajuste en términos generales es alto. Los coeficientes $\beta_{2}$ y $\beta_{3}$ arrojaron desviaciones bajas, sin embargo para el $\beta_{1}$ la desviación fue alta (de casi 66), debido a la altas dispersión de los datos. Dicha dispersión es debida muy probablemente a los gradientes de concentración que presenta la FES (Viccini et al, 2001; Padey et al., 2008). Los coeficientes $\beta_{1}, \beta_{2}$ y $\beta_{3}$, tienen el mismo significado que los explicados para la concentración de proteína; para el caso de la concentración de ergosterol máxima promedio alcanzada, el valor fue de $116,146 \mu \mathrm{g} \mathrm{Ergosterol} / \mathrm{g}$ Sustrato $\pm 65,8616$. Es importante resaltar que estas curvas se muestran aun en una fase de crecimiento, es decir no llegaron a la fase estacionaria en los 30 días de evaluación. Con los valores promedios obtenidos en los coeficientes del modelo logístico, se obtiene las curvas modeladas de biomasa para la concentraciones de proteína y ergosterol en función del tiempo y sus resultados se muestran en la figura 3.
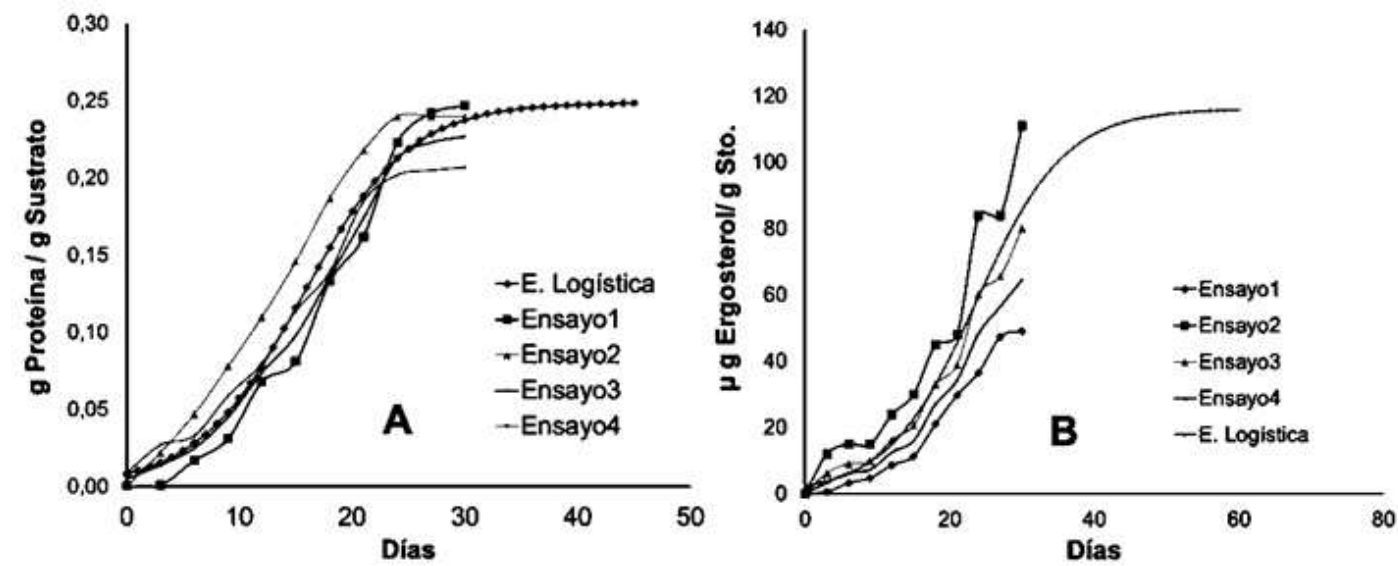

Fig. 3. Curvas de biomasa modeladas mediante la ecuación logística promedio. A. Concentración de proteína vs tiempo. B. Concentración de ergosterol vs tiempo

En la figura 3, aparecen los 4 ensayos de la curva de biomasa obtenidos experimentalmente y la curva obtenida mediante la ecuación logística modelada, en forma de S, tal como se describió anteriormente, sin fase lag o de adaptación como lo describe Vicchini y colaboradores (2001), propia del comportamiento 
logístico. La ecuación logística es un modelo no estructurado, empírico y descriptivo, obtenido con datos experimentales, que a pesar de su simplicidad matemática, puede dar una adecuada aproximación del crecimiento celular. El modelo logístico, se ha empleado en FES para modelar el crecimiento de varios organismos tales como basidiomicetos del genero Pleurotus (Telles et al., 2008; Sarikaya et al., 1997), con hongos filamentosos, como Mucor bacilliformis (Lareo, 2006) y Aspergillus niger (Ali et al., 2009; Hamidi et al., 2004) y Trichoderma reesei (Smits et al., 1999). También se ha empleado para el modelado de la levadura Kluyveromyces marxianus (Mazutti et al., 2010).

Adicional a los datos analizados ensayo por ensayo, se llevó a cabo un ajuste con todos los datos obtenidos experimentalmente en función del tiempo para la concentración de proteína y para la concentración de ergosterol. Dicho ajuste se llevó a cabo en el CFTOOL de Matlab; la figura 4 muestra el ajuste de todos los datos obtenidos en función del tiempo.

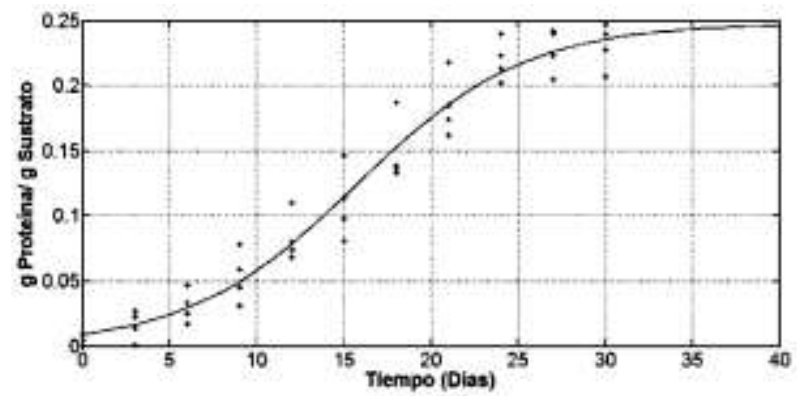

A

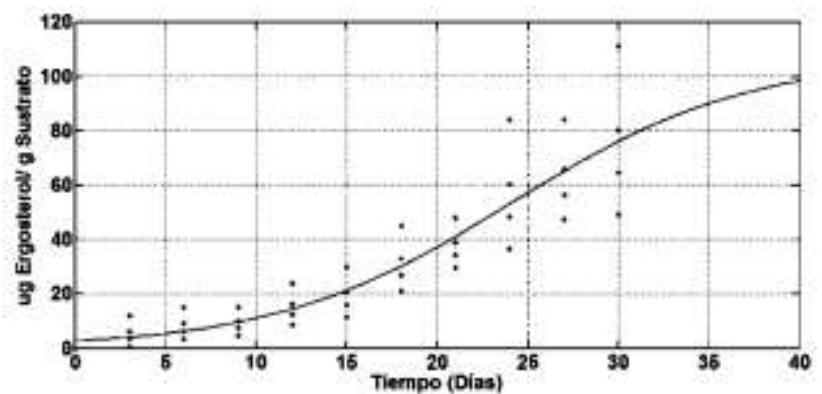

B

Fig. 4. Curvas de biomasa con todos los datos analizados en el CFTOOL. A. Concentración de proteína vs tiempo. B. Concentración de ergosterol vs tiempo.

En la tabla 3, aparecen resumidos los coeficientes obtenidos con su respectivo $r^{2}$ ajustado. Estos resultados se validaron con el programa $R$, obteniendo resultados similares. En la tabla 4 , se muestra la comparación de los coeficientes de la ecuación logística para los resultados obtenidos ensayo por ensayo y para todos los datos.

Tabla 3. Coeficientes del modelo logístico arrojados por el CFTOOL para los datos en conjunto de la concentración de proteína vs tiempo y el contenido de ergosterol vs tiempo.

\begin{tabular}{|c|c|c|}
\hline $\begin{array}{c}\text { Coeficientes } \\
\text { CFTOOL }\end{array}$ & $\begin{array}{c}\text { Datos Concentración } \\
\text { Proteína }\end{array}$ & $\begin{array}{c}\text { Datos Concentración } \\
\text { Ergosterol }\end{array}$ \\
\hline$\beta_{1}(\mathrm{~g}$ Proteína/ g Sto.) & 0,284 & 107,000 \\
\hline$\beta_{2}$ & 3,251 & 3,681 \\
\hline$\beta_{3}\left(\right.$ Dias $\left.^{-1}\right)$ & $-0,207$ & $-0,153$ \\
\hline$r^{2}$ Ajustado & 0,958 & 0,828 \\
\hline
\end{tabular}

Tabla 4. Comparación de los coeficientes obtenidos ensayo por ensayo y todos los datos en conjunto

\begin{tabular}{|c|c|c|c|c|}
\hline Coeficientes & $\begin{array}{c}\text { Concentración de proteína } \\
\text { (Ensayo por Ensayo) }\end{array}$ & $\begin{array}{c}\text { Concentración de } \\
\text { proteína (Datos } \\
\text { Completos) }\end{array}$ & $\begin{array}{c}\text { Concentración de } \\
\text { ergosterol (Ensayo por } \\
\text { Ensayo) }\end{array}$ & $\begin{array}{c}\text { Concentración de } \\
\text { ergosterol (Datos } \\
\text { Completos) }\end{array}$ \\
\hline$\beta_{1}$ (g Proteína/g Sto.) & 0,2489 & 0,2841 & 116,146 & 107 \\
\hline$\beta_{2}$ & 3,3378 & 3,251 & 3,8661 & 3,681 \\
\hline$\beta_{3}$ (Dias $^{-1}$ ) & $-0,2131$ & $-0,2069$ & $-0,1633$ & $-0,153$ \\
\hline $\mathrm{r}^{2}$ Ajustado & 0,9937 & 0,9583 & 0,9872 & 0,828 \\
\hline
\end{tabular}

La tabla 4 muestra los resultados obtenidos de los parámetros, siendo similares en ambos modelos, sobre todo para la concentración de proteína; en el caso del ergosterol, la mayor diferencia está en el coeficiente $\beta_{1}$. Los ajustes individuales muestran valores de $r^{2}$ bastante buenos, y solo el ergosterol muestra un coeficiente menor de 0,9. De otro lado, se obtuvo una modelación con los datos promedio y los datos individuales, tanto para la concentración de proteína como para la concentración de ergosterol. Los comportamientos de dichas modelaciones se muestran en la figura 5. 

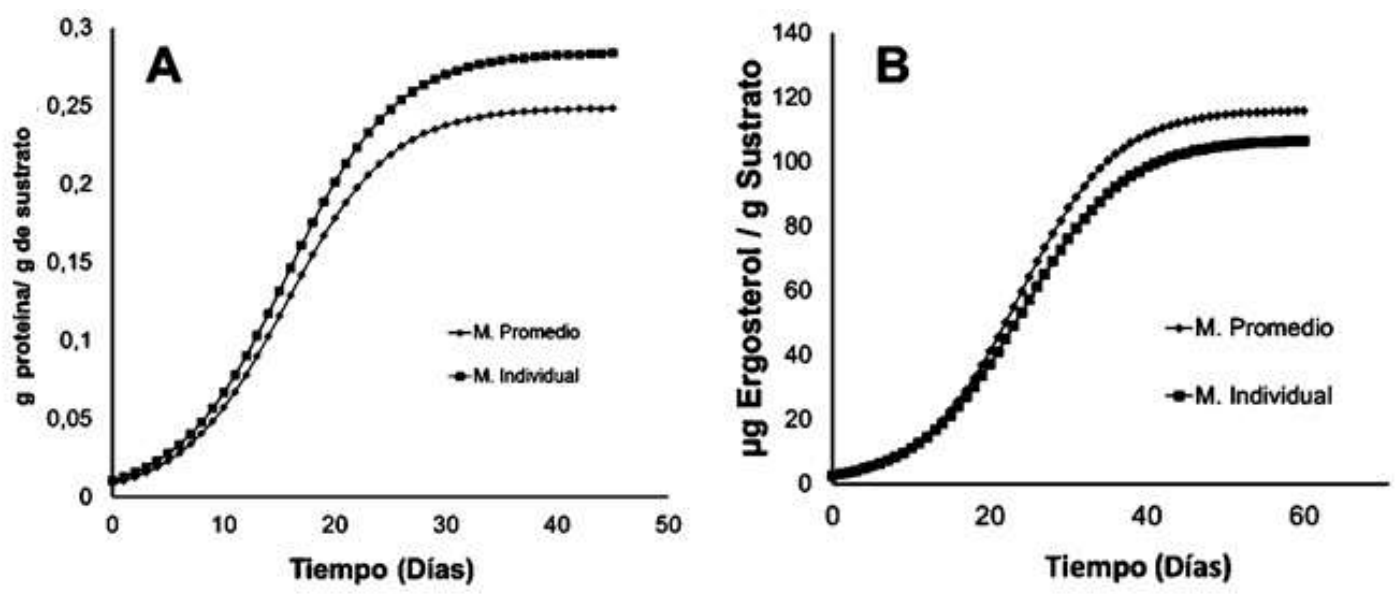

Fig. 5. Curvas de biomasa modeladas para los datos analizados en ensayo por ensayo y todos los datos juntos. A. Concentración de proteína vs tiempo. B. Contenido de ergosterol vs tiempo

De acuerdo con la figura 5, se muestra que para el caso de la modelación con la proteína (A) el modelo ensayo por ensayo está por encima del modelo con todos los datos; en el caso de la modelación del ergosterol (B), el modelo de todos los datos se encuentra por encima al modelo ensayo por ensayo. Comparando los coeficientes obtenidos en el modelo para cada uno de los ensayos, con el de todos los datos, se puede observar que en este caso el ajuste del $r^{2}$ ajustado disminuye.

En el caso de la concentración de proteína el $\mathrm{r}^{2}$ es de 0,9583 y para el contenido de ergosterol es $\mathrm{r}^{2}$ es de 0,828 , los cuales fueron menores al $r^{2}$ para cada uno de los ensayos o modelo individual. Esta disminución es probablemente debida a la dispersión de los datos, propia del fenómeno de la FES, como lo han explicado autores como Mazutti et al (2010), Padey et al (2008), Viccini et al (2001) y como se había mencionado anteriormente. A pesar de que estos $r^{2}$ son inferiores para todos los datos, se pueden emplear para modelar de forma apropiada el crecimiento del Pleurotus pulmonarius en tusa de maíz. De Jager et al (2009), obtuvieron para una FES $r^{2}$ entre 0,89 y 0,75 para el modelo logístico, los cuales son inferiores a los obtenidos en este trabajo. Desde el punto de vista estadístico el modelo obtenido con todos los datos sería el que más se ajustaría; el otro modelo, de los datos individuales, es un resumen de los modelos particulares.

\section{Resultados con las RNA}

En una red neuronal, las neuronas se pueden conectar en capas y organizarse en forma paralela para el procesamiento, creando una red. Esta red puede tener un número de capas y neuronas variables según lo requiera el problema específico; el algoritmo de aprendizaje busca obtener los denominados pesos y umbrales que minimicen el error con respecto a los datos originales. Es un concepto similar al ajuste de mínimos cuadrados en la regresión lineal usada ampliamente en estadística (Haykin, 2005). Para este caso de varias capas y varias neuronas, se obtienen una ecuación matemática de salida por cada capa de forma matricial, dada por la ecuación 2:

$\bar{Y}=\bar{\Phi}(\mathbf{W} * \overline{\mathrm{X}}+\overline{\mathrm{b}})$

Donde $\bar{Y}$ es el vector de salida de la capa. $\bar{\Phi}$, es la función vectorial de dimensión (número de neuronas) con la función de activación respectiva. $W$, es la matriz de pesos sinápticos de $\mathrm{m}$ filas y $\mathrm{n}$ columnas, siendo $\mathrm{m}$ el número de neuronas y $\mathrm{n}$ el número de entradas de la capa anterior. $\overline{\mathrm{x}}$, es el vector de dimensión $\mathrm{n}$ con los datos de entrada y $\bar{b}$, el vector de umbrales (bias) de dimensión $\mathrm{m}$. Con lo anterior, entrenando la red neuronal con el NFTOOL, se obtuvieron los parámetros como se muestran en la figura 6.

Como criterio del análisis del error, se empleó la sumatoria media del error cuadrático (RMSE, por sus iniciales en inglés) los cuales aparecen en la tabla 5, donde se compararan con los obtenidos por el modelo logístico. Con la red neuronal entrenada, se obtuvieron los modelos de la figura 7. 


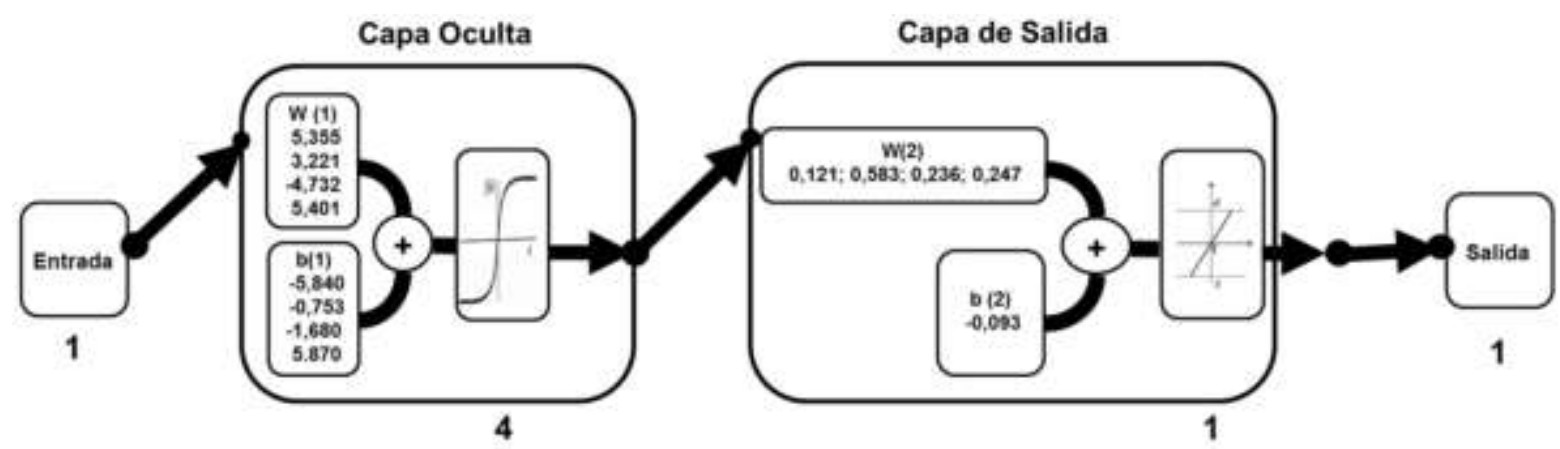

A

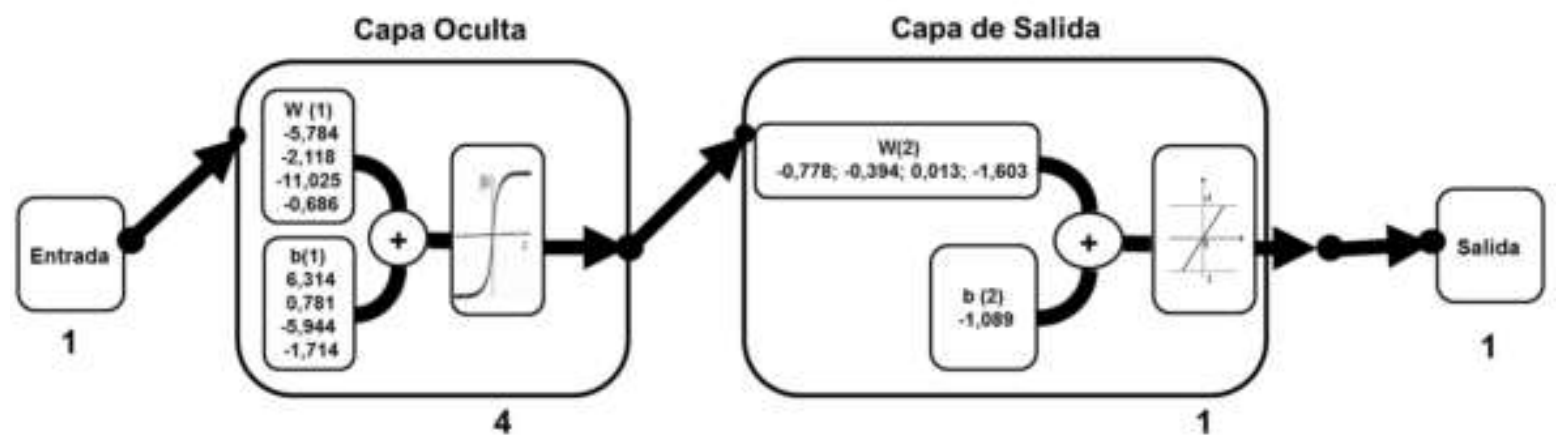

B

Fig. 6. RNA obtenidas con el NFTOOL para el conjunto de datos. A. Concentración de proteína. B. Concentración de ergosterol.
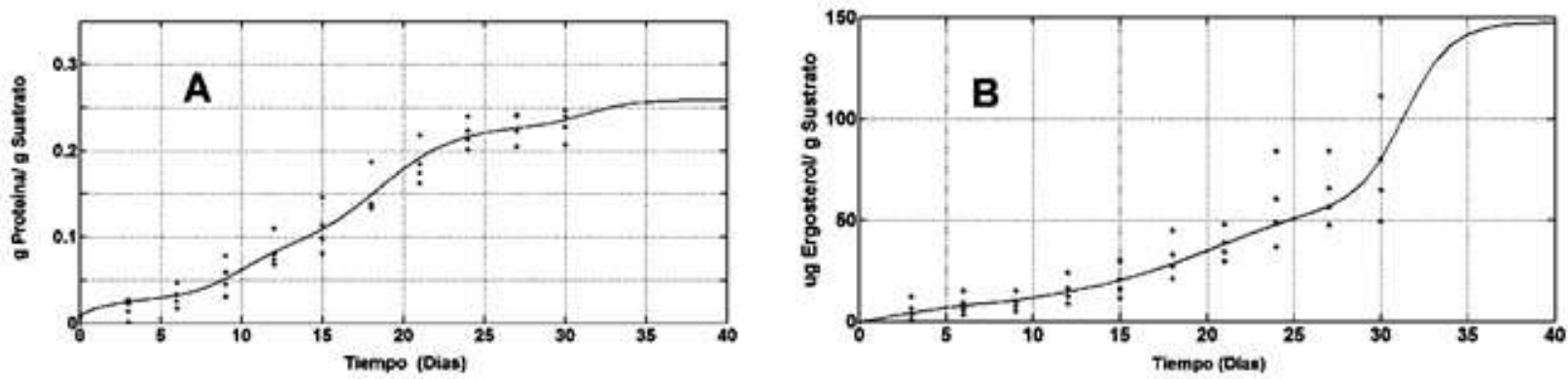

Fig. 7. Ajuste de las curvas de biomasa en función del tiempo empleando RNA. A. Concentración de proteína vs tiempo. B. Concentración de ergosterol vs tiempo.

Tabla 5. Comparación de los RMSE obtenidos por las RNA y el modelo logístico para la concentración de proteína y la concentración de ergosterol en función del tiempo

\begin{tabular}{|c|c|c|c|c|}
\hline \multirow{2}{*}{ RMSE } & $\begin{array}{c}\text { RNA concentración } \\
\text { de proteína }\end{array}$ & $\begin{array}{c}\text { M. Logístico } \\
\text { conc. proteína }\end{array}$ & $\begin{array}{c}\text { RNA concentración } \\
\text { de ergosterol }\end{array}$ & $\begin{array}{c}\text { M. Logístico } \\
\text { conc. Ergosterol }\end{array}$ \\
\cline { 2 - 5 } & 0,017 & 0,176 & 11,394 & 11,43 \\
\hline
\end{tabular}

Comparando los resultados del RMSE mediante las RNA y el modelo logístico para la concentración de proteína, se pueden observar que estos valores son muy similares de la misma manera si se compara el RMSE para la concentración de ergosterol lo cual indica que los dos métodos podrían predecir el comportamiento del crecimiento para el Pleurotus pulmonarius en FES empleando tusa de maíz como sustrato. Tal como se mencionó en la introducción, uno de los grandes inconvenientes y retos que enfrenta la FES es la medición y modelación del crecimiento; las RNA aparecen como herramientas para modelar el crecimiento en FES, sobre todo por lo complicado que es el fenómeno o cuando poco se sabe del mismo, tal como sucedió al inicio de este trabajo (Thomas et al., 2013; Zhang et al., 2012 Lenz et al., 2004). Ejemplo de implementación de RNA en fermentaciones es el trabajo realizado por Desai y colaboradores (2008) arrojando valores de RMSE de 0,11 y 0,73. 
De igual forma que con el modelo logístico, también se analizaron los datos ensayo por ensayo tanto para la concentración de proteína como para la concentración de ergosterol. En la figura 8, se muestran dichos resultados.

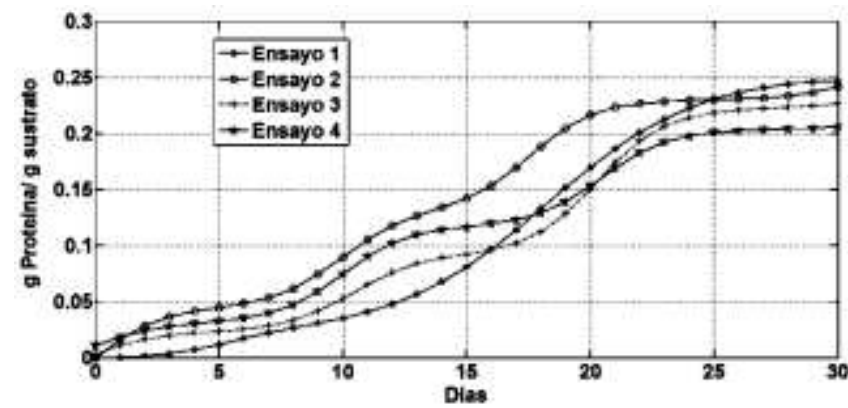

A

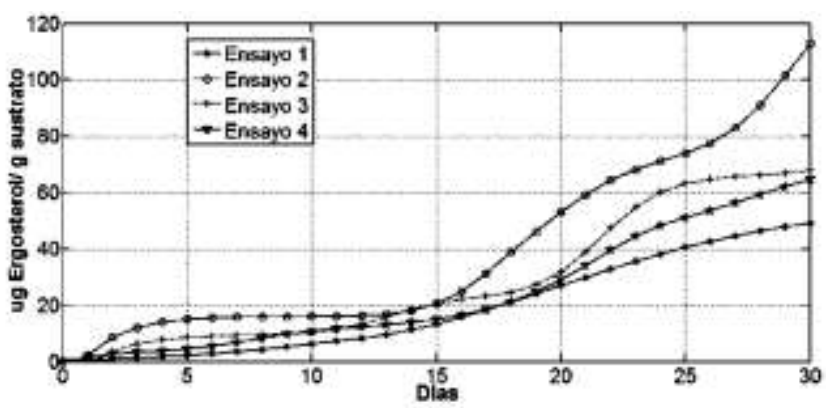

B

Fig. 8. Ajuste de las curvas de biomasa en función del tiempo empleando RNA de forma individual. A.

Concentración de proteína vs tiempo. B. Concentración de ergosterol vs tiempo

La tabla 6, resume los RMSE para cada uno de los ensayos de la concentración de proteína y concentración de ergosterol tanto para el modelo logístico como para las RNA

Tabla 6. Comparación de los RMSE obtenidos por las RNA y el modelo logístico para las curvas de crecimiento empleando concentración de proteína y concentración de ergosterol ensayo por ensayo

\begin{tabular}{|c|c|c|c|c|}
\hline \multirow{2}{*}{ ENSAYO } & \multicolumn{4}{|c|}{ R. M. S. E. } \\
\cline { 2 - 5 } & $\begin{array}{c}\text { RNA } \\
\text { C. Proteína }\end{array}$ & $\begin{array}{c}\text { Modelo logístico } \\
\text { C. Proteína }\end{array}$ & $\begin{array}{c}\text { RNA } \\
\text { C. Ergosterol }\end{array}$ & $\begin{array}{c}\text { Modelo logístico } \\
\text { C. Ergosterol }\end{array}$ \\
\hline Ensayo 1 & 0,0960 & 0,0079 & 1,1953 & 1,1254 \\
\hline Ensayo 2 & 0,0680 & 0,0048 & 6,8728 & 5,3641 \\
\hline Ensayo 3 & 0,0760 & 0,0046 & 4,7330 & 2,5047 \\
\hline Ensayo 4 & 0,0159 & 0,0059 & 1,8794 & 1,2492 \\
\hline Promedio & 0,0640 & 0,0058 & 3,6701 & 2,5608 \\
\hline
\end{tabular}

De la tabla 6 se puede observar que los datos analizados para las RNA en la concentración de proteína, los RMSE individuales de los modelos logísticos fueron aproximadamente 11 veces menores que para las RNA. Para la concentración de ergosterol, también el RMSE promedio del modelo logístico fue menor, esta vez en 1.43 veces, una diferencia menor comparada con la encontrada para la concentración de proteína. Cabe mencionar que se usaron redes neuronales de igual tamaño tanto para le concentración de proteína como para la concentración de ergosterol; sin embargo, es posible aumentar el número de neuronas, si se quiere un mejor ajuste (Haykin, 2005), sobre todo para el de caso de la proteína donde las RNA arrojaron un RMSE aproximadamente 11 veces mayor que el obtenido por el modelo logístico. Karakuzu et al., (2006) emplearon la red neuronal feed forwad (backpropagation) en un proceso de fermentación, para la estimación del crecimiento de Saccharomyces cerevisiae en un proceso por lote alimentado y encontraron un buen ajuste a su modelo experimental; de esta manera se corrobora que las RNA pueden ser empleadas para modelar bioprocesos.

\section{CONCLUSIONES}

Se pudo realizar un ajuste de la cinética del crecimiento indirecto de la biomasa del Pleurotus pulmonarius en tusa de maíz, empleando el modelo logístico y las RNA. Los resultados de la medición de concentración de proteína y ergosterol en función del tiempo, se ajustaron bien a los modelos empleados. Desde el punto de vista estadístico, los modelos obtenidos ensayo por ensayo son un resumen de los modelos particulares, mientras que el de todos los datos, se ajusta mejor a los resultados que se obtienen por mínimos cuadrados. Para la concentración de proteína, el modelo logístico, obtuvo $\mathrm{R}^{2}$ ajustados superiores a 0,95 . Para la concentración de ergosterol el ajuste de las curvas fue mayor a 0,8. Las RNA arrojaron buenos RMSE, los cuales podrían mejorar tanto para la concentración de proteína como para la concentración de ergosterol aumentando el número de neuronas en la capa oculta a expensas de un mayor número de parámetros. Con los datos del modelo logístico y los RMSE obtenidos para la concentración de proteína, se puede modelar de forma adecuada el crecimiento del Pleurotus pulmonarius en tusa de maíz. 


\section{AGRADECIMIENTOS}

Los autores expresan su agradecimiento al Centro de Investigación para el Desarrollo y la Innovación (CIDI), perteneciente a la Universidad Pontificia Bolivariana, por su apoyo en la financiación del proyecto con número de radicado 255A-06/08-25 del que se derivó esta publicación.

\section{REFERENCIAS}

Ali, M y Mahmoodzadeh, B. Modeling of temperature gradients in packed-bed solid-state bioreactors. Chemical Engineering and Processing: Process Intensification. Volume 48, Issue 1. Pages 446-451 (2009)

Braaksma, A. Schaap, D. J. Protein analysis of the common mushroom Agaricus bisporus. Postharvest Biology and Technology Volume 7, 119-127 (1996)

Brodie, E. Edwards, S. Clipson, N. Soil fungal community structure in a temperate upland grassland soil. FEMS Microbiol Ecol. Jul 1;45(2):105-14 (2003)

Carabajal, M. Levin, L. Albertó, E. Lechner, B. Effect of co-cultivation of two Pleurotus species on lignocellulolytic enzyme production and mushroom fructification. International Biodeterioration \& Biodegradation 66 71-76 (2012).

De Jager, D. Sheldon, M. Edwars, W. Modelling growth kinetics of Streptomyces coelicolor A3(2) in a pressurized membrane gradostat reactor (MGR). Enzyme and Microbial Technology 45 449-456 (2009)

Elangovan, R, L. Philip, K. Chandraraj. Biosorption of chromium species by aquatic weeds: Kinetics and mechanis studies. Journal of Hazardous Materials: 152, 100-112 (2008).

Hamidi, Z. Shojaosadati, S. Rinzema, A. Modelling of simultaneous effect of moisture and temperature on A. niger growth in solid-state fermentation. Biochemical Engineering Journal. Volume 21, Issue 3. Pages 265$272(2004)$

Hashemi, M. Razavi, H. Shojoasadati, A. Mousavi, S. Khajeh, S. Safari, M. Mathematical modeling of biomass and $\alpha$-amylase production kinetics by Bacillus $s p$. in solid-state fermentation based on solid dry weight variation. Biochemical Engineering Journal 53 159-164 (2011)

Haykin, S. Neural Networks. A comprehensive Foundation. $2^{\text {nd }}$ Edition. $224-225$. Pearson Prentice Hall. (2005)

Hölter, U. Lenz, J. Solid-state fermentation - Are there any biotechnological advantages?. Current Opinion in Microbiology. 8, 301-306 (2005).

Jenzsch, M., Simutis, R., Lübbert, A. Optimization and Control of Industrial Microbial Cultivation Processes. Eng. Life Sci . 6 (2). 117-124 (2006).

Karakuzu, C. Turker, M. Ozturk, S. Modelling, on-line state estimation and fuzzy control of production scale fed-batch baker's yeast fermentation. Control Engineering Practice 14 959-974 (2006)

Khaouane, L,. Benkortbi, O, Hanini, S. Si-Moussa, C. Modeling of an industrial process of pleuromutilin fermentation using feed-forward neural networks. Brazilian Journal of Chemical Engineering. Vol. 30, No. 01, pp. 105 - 116, January - March, (2013)

Lareo, C. Sposito, A. Bossio, A. Volpe, D. Characterization of growth and sporulation of Mucor bacilliformis in solid state fermentation on an inert support. Enzyme and Microbial Technology. Volume 38, Issues 3-4, 391-399 (2006).

Lenz, J. Höfer, H. Krasenbrink, J. Hölker, U. A survey of computational and physical methods applied to solid-state fermentation. Appl Microbiol Biotechnol 65, 9-17 (2004)

Mazutti, $\mathrm{M}$ y otros ocho autores. Mathematical modeling of Kluyveromyces marxianus growth in solid-state fermentation using a packed-bed biorreactor. 37, 391- 400 (2010)

Mitchell, D. Krieger, N. Berovic, M. Solid state fermentation biorreactors. Fundamentals of design and operation. New York, Springer Science Business Media. 481, (2006) 
Mitchell, D. Von Meien, O. Krieger, N. Diba, Dalsenter, F. A review of recent developments in modeling of microbial growth kinetics and intraparticle phenomena in solid-state fermentation. Biochemical Engineering Journal 17 15-26 (2004)

Muller, M. Souza, A. Dal'Boit, S. Mitchell, D. Thermal denaturation: is solid-state fermentation really a good technology for production of enzymes?. Bioresource Technology. 93 261-268 (2004).

Muñoz, R. Llano, J. Saez, L. Relación entre el Parque de Tractores Agrícolas y el Patrón de Difusión y Adopción mediante un Modelo Logístico. Información Tecnológica, 22(6), 121-128 (2011)

Orlandoni , G. Modelos de crecimiento de poblaciones biológicas: Un enfoque de dinámica de sistemas. Economía- XXII, 13 pp. 115-146 (1997).

Oviedo, J y otros cuatro autores. Tusa de maíz enriquecida con micelio de basidiomicetos (Pleurotus pulmonarius) para alimentación animal. Actas del $10^{\circ}$ Concgreso Nacional de Ciencia y Tecnología de Alimentos - ACTA, en simultánea con el XVI Seminario Latinoamericano de Ciencia y Tecnología de Alimentos - ALACCTA y $2^{\circ}$ Simposio Latinoamericano de Inocuidad de Alimentos IAFP. ISSN 1909-0846. 21 al 24 de septiembre, Bogotá (2010)

Padey, A. Soccol, C. Larroche, C. Current Developments in Solid-state Fermentations (Editors). Asiatech Publishers, INC (Springer), New Delhi, 3-12 (2008).

Peleg, M. Corradni, M. Normand, M. The logistic (Verhulst) model for sigmoid microbial growth curves revisited. Food Research International, 40, 808-818 (2007)

Prabhakar, A. Krishnaiah, K. Janaun, J. Bono, A. An overview of engineering aspects of solid state fermentation. Malalaysian Journal of Microbiology. 1 (2), 10-16 (2005)

Quiñones y Lecompte. Modelos exponencial y logístico de la población en el Suroeste de Puerto Rico. Revista de investigación $360^{\circ}$ en Ciencias y Matemáticas. Marzo, (I) 3 (2007)

Rani, R. Kumar, A. Soccol, C. Pandey, A. Recent advances in solid state fermentation. Biochemical Engineering Journal. 44 13-18 (2009)

Ribeiro, R. Ergosterol para a determinação de biomassa dos fungos Lentinus crinitus Berk. e Psilocybe castanella Peck em biorremediação do solos. Dissertação (Mestrado) - Universidade Estadual Paulista, Instituto de Biociências de Rio Claro 108 p (2004)

Robinson, T. y Nigam, P. Biorreactor design for protein enrichment of agricultural residues by solid state fermentation. Biochemical Engineering Journal. 13, 197-203 (2003)

Rodriguez, C y Sanroman, A. Application of solid state fermentation to food industry. A review. Journal of Food Engineering. 76, 291-302 (2006).

Sarikaya, A y Ladishc, M. An unstructured mathematical model for growth of Pleurotus ostreatus on lignocellulosic material in solid-state fermentation systems. Applied Biochemistry and Biotechnology. Volume 62, Issue 1, 71-85 (1997)

Smiths, $\mathrm{J}$ y otros seis autores. Modelling fungal solid-state fermentation: the role of inactivation kinetics. Bioprocess Engineering 20, 391-404 (1999)

Spier, M. Letti, L. Woiciechowski, A. Soccol, C. A simplified model for A. Niger FS3 growth during phytase formation in solid State fermentation. Braz. arch. biol. technol. 52, 151-158 (2009)

Sturm, W. Soccolo, C. Amaral, D. Rodriguez, J. Navarro, D. Informatics in Solid State Fermentation. In Current Developments in Solid-state Fermentations (Editors). Asiatech Publishers, INC (Springer), New Delhi, 168-179 (2008).

Téllez, M y Fernandez, F. Growth and laccase production by Pleurotus ostreatus in submerged and solidstate fermentation. Appl Microbiol Biotechnol. 81, 675-679 (2008).

Thomas, L. Larroche, C. Pandey, A. Current developments in solid-state fermentation. Biochemical Engineering Journal, 81, 146-161 (2013). 
Viccini, G y otros ocho autores. Analysis of Growth Kinetic Profiles in SS Fermentation. Food Technol. Biotechnol, 39 (4), 271-294 (2001)

Villada, F. Muñoz, N. García, E. Aplicación de las Redes Neuronales al Pronóstico de Precios en el Mercado de Valores. Información Tecnológica, (23) 4, 11-20 (2012)

Zhang, $\mathrm{Y}$ y otros cinco autores. Artificial Intelligence Techniques to Optimize the EDC/NHS-Mediated Immobilization of Cellulase on Eudragit L-100. International Journal of Molecular Sciences. 13, 7952-7962 (2012) 